\title{
COMPARISON BETWEEN THE AMERICAN JOINT COMMITTEE ON CANCER (AJCC) ANATOMIC AND PROGNOSTIC STAGES FOR BREAST CANCER
}

Mariana Soares Cardoso', Marcelo Antonini Matheus de Paula Solino

${ }^{1}$ Instituto de Assistência Médica ao Servidor Público Estadual - São Paulo (SP), Brazil.

Introduction: The knowledge regarding the biology of breast cancer has grown substantially and resulted in the identification of different breast cancer subtypes based on their molecular profile, which led to an important change in treatment, from a standardized therapy to a personalized one. A panel of experts and AJCC representatives were responsible for preparing the most recent Cancer Staging Manual. The panel recognized the clinical usefulness of biological factors such as histological grade, expression of hormone receptors (HR; estrogen and progesterone) and overexpression and/or amplification of the human epidermal growth factor receptor 2 (HER2) in predicting patient survival ${ }^{7}$ and incorporated data regarding these biomarkers in the new staging system. In addition, for eligible cases, the 'Recurrence score' was also incorporated, generated by the analysis of OncotypeDx (genomic test). The new manual, therefore, started to use 3 stagings. Anatomical staging - based on the classic TNM; clinical prognostic staging and pathological prognosis - association of TNM with prognostic biomarkers (using clinical data in the first and data after surgical treatment in the second). Objective: To verify the agreement between anatomical staging from the $7^{\text {th }}$ edition of the AJCC manual and the prognosis from its $8^{\text {th }}$ edition in a cohort of breast cancer patients at the Hospital do Servidor Público Estadual de São Paulo. Methodology: Observational and cross-sectional study, which evaluated patients undergoing surgical treatment at Hospital do Servidor Público Estadual from March, 2014 to March, 2019. Information was collected regarding age, menopausal status, tumor characteristics, anatomical and clinical staging, neoadjuvant chemotherapy, adjuvant chemotherapy and radiotherapy, and type of surgery performed. Patients were staged using the digital platform "TNM8 Breast Cancer Calculator". Results: 805 patients were included in the analysis. All patients were females aged between 29 and 97 years, mostly in the post-menopausal period (78.88\%). $74.04 \%$ of cases were positive for ER, 66.21\% PR-positive, and 88.07\% HER2negative. Prognostic staging downgraded a total of 285 out of 805 patients (35.4\%). Almost all of the cases that decreased in staging were ER and/or PR+ (283 of 285). Most of those who went up were Triple Negatives (100 out of 111). Conclusion: Prognostic Staging changes the staging in almost half of the cases and there was a greater number of decreased staging in total and an association of increased staging with tumors considered to have a worse prognosis, which is in agreement with several studies already carried out since the new manual came out. 